Pacific Journal of Mathematics

CENTRALIZERS AND $H^{*}$-ALGEBRAS 


\section{CENTRALIZERS AND $H^{*}$-ALGEBRAS}

\section{N. KeLloGG}

A mapping $T$ from a Banach algebra $X$ into itself shall be called a centralizer of $X$ if $x(T y)=(T x) y$ for all $x, y \in X$. A bounded linear operator, $T$, in $X$ shall be called a right [left] centralizer if $T(x y)=(T x) y[T(x y)=x(T y)]$. We show that the space of centralizers forms a closed commutative subalgebra of the bounded linear operators in $X$. The intersection of the space of right centralizers with the space of left centralizers is precisely the algebra of centralizers.

We show that the algebra of right [left] centralizers of an $H^{*}$-algebra is the $W^{*}$-algebra generated by the left [right] multiplication operators and that the commutant of the algebra of right [left] centralizers is the algebra of left [right] centralizers. In order to do this, we construct a net, $\left\{e_{a}\right\}_{a} \in_{D}$ in the $H^{*}$-algebra such that $\left\{e_{a} x\right\}_{a} \in_{D}$ and $\left\{x e_{a}^{*}\right\}_{a} \in D$ converge to $x$. We show that the algebra of centralizers of a commutative $H^{*}$ algebra is the space of bounded functions on a discrete set. Characterizations are given for compact and projection centralizers.

We also study commutative $H^{*}$-algebras in which the irreducible self-adjoint idempotents all have the same norm. We show that two such $H^{*}$-algebras are topologically and algebraically equivalent if and only if they have the same Hilbert space dimension.

The notion of a centralizer was first introduced by Wendel [6] in his work on noncommutative group algebras. Operators similar to centralizers have been studied by Wang [5] in the context of a commutative Banach algebra.

2. Preliminaries. This section is devoted to the necessary definitions and notations. We also prove a very straightforward generalization of several of the results found in Section 2 of [5].

Definition 1. If $X$ is a Banach algebra (complex), then a mapping $T$ from $X$ into $X$ will be called a centralizer of $X$ if $T$ satisfies the identity $x(T y)=(T x) y$, and $\mathscr{C}(X)$ will denote the set of all centralizers of $X$.

Definition 2. If $X$ is Banach algebra and $T$ is a bounded linear

Received January 25. 1965. The results of this paper form part of the author's doctoral dissertation (Louisiana State University, 1964). The research was supported in part by National Science Foundation Grant No. GP-1449 and by the United States Air Force, AFSOR 68-83. 
operator in $X$, then $T$ will be called a right [left] centralizer of $X$ if $T$ satisfies the identity $T(x y)=(T x) y[T(x y)=x(T y)]$. We will use the symbol $R(X)[L(X)]$ to denote the collection of all right [left] centralizers of $X,[6]$.

Definition 3. A Banach algebra $X$ is said to be without order if $X y=(0)$ implies that $y=0$ and $y X=(0)$ implies that $y=0$.

The symbol $B(X)$ will be used to denote the collection of bounded linear operators in $X$ and the operator norm on $B(X)$ will be denoted by $\|\cdot\|_{0}$.

THEOREM 2.1. If $X$ is a Banach algebra which is without order, then $\mathscr{C}(X)$ is a closed commutative subalgebra of $B(X)$ which contains the identity operator.

Proof. We will first show that $\mathscr{C}(X) \subset B(X)$. If $T \in \mathscr{C}(X), x, y$, $z, \in X$ and $a$ and $b$ complex numbers, then

$$
x[T(a y+b z)]=(T x)(a y+b z)=x a(T y)+x b(T z)=x[a T y+b T z] .
$$

Since $X$ is without order, $T(a y+b z)=a T y+b T z$ and thus $T$ is linear. Further, if $y, z \in X$ and $\left\{y_{n}\right\}_{n=1}^{\infty}$ is a sequence in $X$ such that $\left\|y_{n}-y\right\| \rightarrow 0$ and $\left\|T y_{n}-z\right\| \rightarrow 0$, then

$$
\|x z-x(T y)\| \leqq\|x\|\left\|z-T y_{n}\right\|+\|T x\|\left\|y_{n}-y\right\|
$$

for each $x \in X$. Therefore $x z=x(T y)$ and $X$ without order implies that $z=T y$. We now apply the Closed Graph Theorem to conclude that $T$ is bounded and hence $\mathscr{C}(X) \subset B(X)$. It is easy to see that $R(X) \cap L(X)=\mathscr{C}(X)$ and hence $[(T S) x] y=T[(S x) y]=T[S(x y)]=$ $(T S)(x y)$ which implies that $T S \in R(X)$ whenever $T, S \in \mathscr{C}(X)$. Similarly, $T S \in L(X)$ and thus $T S \in R(X) \cap L(X)=\mathscr{C}(X)$. It is obvious that $\mathscr{C}(X)$ is a linear space which is closed under scalar multiplication and hence $\mathscr{C}(X)$ is a subalgebra of $B(X)$. If $T, S \in \mathscr{C}(X)$, then

$$
x[(T S) y]=x[T(S y)]=(T x)(S y)=[S(T x)] y=[(S T) x] y=x[(S T) y] .
$$

Since $X$ is without order, we conclude that $(T S) y=(S T) y$ and that $\mathscr{C}(X)$ is commutative. The identity operator is clearly an element of $\mathscr{C}(X)$. If $\left\{T_{n}\right\}_{n=1}^{\infty}$ is a sequence in $\mathscr{C}(X)$ which is Cauchy with respect to the operator norm, then since $\mathscr{C}(X) \subset B(X)$, there exists $T \in B(X)$ such that $\left\|T_{n}-T\right\|_{0} \rightarrow 0$. If $x, y \in X$, then

$$
\begin{aligned}
\|x(T y)-(T x) y\| & \leqq\left\|x(T y)-x\left(T_{n} y\right)\right\|+\left\|\left(T_{n} x\right) y-(T x) y\right\| \\
& \leqq 2\|x\|\|y\|\left\|T_{n}-T\right\|_{0}
\end{aligned}
$$


which converges to zero with $n$. Hence $x(T y)=(T x) y$ and $T \in \mathscr{C}(X)$. Therefore $\mathscr{C}(X)$ is closed with respect to the operator norm and the proof of the theorem is complete.

3. Centralizers of $H^{*}$-algebras. Throughout this section, $H$ will denote an $H^{*}$-algebra. We will study the spaces $R(H)$ and $L(H)$ in considerable detail and note that it is not hard to show that $R(H)$ and $L(H)$ are $C^{*}$-algebras (hence $B^{*}$-algebras) each of which contains the identity operator.

Theorem 3.1. Each of $R(H)$ and $L(H)$ is a $W^{*}$-algebra.

Proof. Since $R(H)$ is a self-adjoint subalgebra of $B(H)$, we must only show that it is weak operator closed. To do this, let $A \in B(H)$ and let $\left\{A_{a}\right\}_{a} \in_{D}$ be a net in $R(H)$ such that $\left\{A_{a}\right\}_{a} \in_{D}$ converges to $A$ in the strong operator topology. Then, for $x, y \in H$, we have

$$
\begin{aligned}
\|A(x y)-(A x) y\| & \leqq\left\|A(x y)-A_{a}(x y)\right\|+\left\|\left(A_{a} x\right) y-(A x) y\right\| \\
& \leqq\left\|A(x y)-A_{a}(x y)\right\|+\left\|A_{a} x-A x\right\|\|y\|
\end{aligned}
$$

which converges to zero with $a$. Hence $A(x y)=(A x) y, A \in R(H)$ and $R(H)$ is strong operator closed. Since $R(H)$ is a self-adjoint subalgebra of $B(H)$, the strong and weak operator closures of $R(H)$ coincide, [2, 448]. Hence $R(H)$ is weak operator closed and thus is a $W^{*}$-algebra. The proof for $L(H)$ is similar and will be omitted.

THEOREM 3.2. There is a net $\left\{e_{a}\right\}_{a} \in_{D}$ contained in $H$ with the property that $\left\{e_{a} x\right\}_{a \in D}$ and $\left\{x e_{a}^{*}\right\}_{a} \in_{D}$ converge to $x$ for every $x \in H$.

Proof. Let $\left\{e_{\alpha}\right\}$ be a maximal family of nonzero mutually orthogonal irreducible self-adjoint idempotents of $H$ and let $D$ be the set of all finite sets of the indices $\alpha$, directed by inclusion. To $a=\left(\alpha_{1}, \alpha_{2}, \cdots, \alpha_{n}\right)$, let $e_{a}=e_{\alpha_{1}}+e_{\alpha_{2}}+\cdots+e_{\alpha_{n}}$ correspond. The net $\left\{e_{a}\right\}_{a} \in_{D}$ clearly satisfies the requirements of the theorem.

The author wishes to express his appreciation to the referee for the above proof.

COROLLARY 3.3. The $W^{*}$-algebra generated by the left [right] multiplication operators is $R(H)[L(H)]$.

Proof. For each $x \in H$, define the left multiplication operator $L_{x}$ in $H$ by $L_{x}(y)=x y$ and let $\mathscr{L}(H)=\left\{L_{x}: x \in H\right\}$. Let $\left\{e_{a}\right\}_{a} \in_{D}$ be the net constructed in Theorem 3.2. For $A \in R(H), L_{\Delta e_{a}}(x)=\left(A e_{a}\right) x=A\left(e_{a} x\right)$ and since $\left\{e_{a} x\right\}_{a} \in_{D}$ converges to $x$, we have that $L_{A e_{a}}$ converges to $A$ 
in the strong operator topology. Thus $\mathscr{L}(H)$ is strong operator dense in $R(H)$ and since $\mathscr{L}(H)$ is a self-adjoint subalgebra of $B(H)$, it follows that the $W^{*}$-algebra generated by $\mathscr{L}(H)$ is $R(H)$. The proof for right multiplication operators is analogous and will be omitted.

For $S$ an arbitrary subset of $B(H)$, denote by $W(S)$ the smallest $W^{*}$-algebra containing $S$ and denote by $S^{\prime}$ the set of all operators in $B(H)$ which commute with all the operators in $S \cup S^{*}$ where $S^{*}=\left\{A^{*}: A \in S\right\}$.

\section{TheoRem 3.4. $R(H)^{\prime}=L(H)$ and $L(H)^{\prime}=R(H)$.}

Proof. It is known, see [2, 445], that if $S$ is any set in $B(H)$, then $S^{\prime}=W(S)^{\prime}$. This fact, together with Corollary 3.3, implies that $R(H)^{\prime}=W(\mathscr{L}(H))^{\prime}=\mathscr{L}(H)^{\prime}$. Now, for $A \in R(H)^{\prime}$, we have that $A \in \mathscr{L}(H)^{\prime}$ and hence $A L_{x}=L_{x} A$ for all $x \in H$. Thus $A(x y)=A\left(L_{x} y\right)=$ $L_{x}(A y)=x(A y), A \in L(H)$ and $R(H)^{\prime} \subset L(H)$. The other containment is trivial and thus $L(H)=R(H)^{\prime}$. Also we have that $R(H)^{\prime \prime}=L(H)^{\prime}$ and since $R(H)$ is a $W^{*}$-algebra containing the identity, $L(H)^{\prime}=$ $R(H),[2,448]$.

REMARK 1. Since $f_{x}(A)=(A x, x)$ is a positive linear functional on $R(H)[L(H)]$ for each $x \in H$, it is easily seen that $R(H)[L(H)]$ is a symmetric and reduced (hence semi-simple) algebra.

REMARK 2. Since each of $R(H)$ and $L(H)$ is a $W^{*}$-algebra with the identity operator as unit, then $\mathscr{C}(H)=R(H) \cap L(H)$ has the same properties.

REMARK 3. Note that $\mathscr{C}(H)$ is also symmetric, reduced and semisimple since it is a closed commutative subalgebra of $B(H)$ containing $I$ and thus is isometric *-algebra isomorphic to the bounded continuous functions on its compact regular maximal ideal space (e.g., see [2, 232]).

4. Centralizers of commutative $H^{*}$-algebras, We will now focus our attention on commutative $H^{*}$-algebras and first give a characterization of the centralizers of a commutative $H^{*}$-algebra as the set of all bounded (continuous) complex-valued functions on a discrete space. In this section $H$ will be a commutative $H^{*}$-algebra.

Let $E=\left\{e_{a} /\left\|e_{a}\right\|: e_{a}\right.$ is an irreducible self-adjoint idempotent $\}$. Note that each minimal ideal of $H$ is the one-dimensional ideal generated by an $e_{a}$. We can now identify $E$ with $\mathfrak{M}_{H}$ (regular maximal ideal space of $H$ ) and if we give $E$ the discrete topology, then $E$ and $\mathfrak{M n}_{H}$ are also topologically equivalent. Note also that $E$ is a complete orthonormal basis for $H$. 
DEFINITION 4. A function $f$ from $\mathfrak{M}_{H}$ into the complex numbers will be called a multiplier of $H$ provided $f \hat{H} \subset \hat{H}$, where $\hat{H}=\{\hat{x}: x \in H\}$, $\widehat{x}$ is the Gelfand transform of $x$ and the set of all multipliers of $H$ will be denoted by $M(H)$.

REMARK 4. It is shown in [5] that $M(H) \subset C\left(\mathfrak{M}_{H}\right)$, the bounded continuous complex-valued functions on $\mathfrak{M}_{H}$, and there is a natural mapping from $\mathscr{C}(H)$ onto $M(H)$ which is a norm-decreasing algebra isomorphism. We will often refer to this mapping as the Wang mapping.

THEOREM 4.1. There exists a *algebra isomorphism which is an isometry from $\mathscr{C}(H)$ onto $C(E)$, the set of all bounded complexvalued functions on the discrete space $E$, where $E$ is as above.

Proof. By our previous remark, there is a mapping from $\mathscr{C}(H)$ onto $M(H)$, a subset of $C\left(\mathfrak{M}_{H}\right)$. By identifying $\mathfrak{M}_{H}$ with $E$, we have $M(H)$ as a subset of $C(E)$. The correspondence between $\mathfrak{M l}_{H}$ and $E$ gives the Gelfand transform the form $\hat{x}(e)=(x, e) /\left\|e_{a}\right\|$, where $e=$ $e_{a} /\left\|e_{a}\right\|$. Recall that the defining equation for the Wang mapping, $\Phi$, is $\Phi(A)(h) \hat{x}(h)=\widehat{A x}(h)$, for all $x \in H$ and $h \in \mathfrak{M}_{H}$. Since $E \subset H$, we have that $\Phi(A)(e)=(A e, e)$ for $A \in \mathscr{C}(H)$ and $e \in E$. If $g \in C(E)$ and $x \in H$, then $z=\sum_{E}(x, e) g(e) e$ is an element of $H$. If $x \in H$, then $g(e) \hat{x}(e)=g(e)(x, e) /\left\|e_{a}\right\|$ and $\widehat{z}(e)=(x, e) g(e) /\left\|e_{a}\right\|$. Therefore $g(e) \hat{x}(e)=$ $\widehat{z}(e)$ and thus $g \hat{H} \subset \hat{H}$, so that $g \in M(H)$. The mapping clearly takes $A^{*}$ into the conjugate of the image of $A$ and thus the only thing remaining is to prove that the Wang mapping is an isometry. For $A \in \mathscr{C}(H)$, we have that $(A e, f)=\left(A\left[\left\|e_{a}\right\| e e\right], f\right)=0$ for $e, f \in E$ and $e \neq f$ and

$$
(A x, e)=\left(A\left[\sum_{f \in E}(x, f) f\right], e\right)=(x, e)(A e, e) .
$$

Therefore

$$
\|A x\|^{2}=\sum_{E}|(A x, e)|^{2}=\sum_{E}|(x, e)|^{2}|(A e, e)|^{2} \leqq\|\Phi(A)\|_{\infty}^{2}\|x\|^{2}
$$

and hence $\|A\|_{0} \leqq\|\Phi(A)\|_{\infty}$, so that $\Phi$ is an isometry. This completes the proof of the theorem.

We will now use the mapping of Theorem 4.1 to characterize the compact operators in $\mathscr{C}(H)$. The proof will use the following lemma which gives a necessary and sufficient condition for a projection operator to be in $\mathscr{C}(H)$.

Lemma 4.2. If $P$ is a projection operator in $H$, then $P \in \mathscr{C}(H)$ if and only if $H=I_{1} \oplus I_{2}$, where $I_{1}$ is an ideal and $I_{2}$ is a subalgebra of $H$ with $P=P_{I_{2}}$ (the projection onto $I_{2}$ ) and $I_{1} I_{2}=(0)$. 
Proof. First, assume that $P \in \mathscr{C}(H)$, let $I_{2}=P(H)$, and let $I_{1}$ be the orthogonal complement of $I_{2}$ in $H$. By the definition of $I_{1}$ and $I_{2}$, $H=I_{1} \oplus I_{2}$. If $x \in I_{1}$ and $y \in H$, then $P(x y)=0$, since $P(x y)=P(x) y$ and $x$ is in the orthogonal complement of the range of $P$. Hence $x y \in I_{1}$ and $I_{1}$ is an ideal of $H$. Furthermore, if $x, y \in I_{2}$, then $P x=x$ and $P(x y)=x y$ which implies that $x y \in I_{2}$ and hence $I_{2}$ is a subalgebra of $H$. If $x \in I_{1}$ and $y \in I_{2}$, then $x y=x(P y)=P(x y)=0$ since $x \in I_{1}$ (an ideal). Thus $I_{1} I_{2}=(0)$. Conversely, if $x, y \in H$, then $x=x_{1}+x_{2}$ and $y=y_{1}+y_{2}$ where $x_{1}, y_{1} \in I_{1}$ and $x_{2}, y_{2} \in I_{2}$. Since $P=P_{I_{2}}, P x=$ $P\left(x_{1}+x_{2}\right)=x_{2}$ and $P y=y_{2}$ and $x_{2} y_{1}=0=y_{2} x_{1}$. Hence $x(P y)=$ $\left(x_{1}+x_{2}\right) y_{2}=x_{2} y_{2},(P x) y=x_{2}\left(y_{1}+y_{2}\right)=x_{2} y_{2}$ and $x(P y)=(P x) y$. Therefore we have $P \in \mathscr{C}(H)$, concluding the proof.

We now introduce some notation to be used in the following theorem. By $I_{0}(H)$, we will denote the set of all compact operators in $H$. We will denote by $C_{0}(E)$ and $C_{\infty}(E)$, respectively, the subspaces of $C(E)$ which are the functions with compact support and the functions which vanish at $\infty$. Let $\mathscr{C}_{\infty}(H)=\Phi^{-1}\left(C_{\infty}(E)\right)$ and $\mathscr{C}_{0}(H)=\Phi^{-1}\left(C_{0}(E)\right)$, where $\Phi$ is the Wang mapping.

THEOREM 4.3. The space of all compact centralizers in $H$ is precisely $\mathscr{C}_{\infty}(H)$.

Proof. If $A \in \mathscr{C}_{0}(H)$, then $\Phi(A) \in C_{0}(E)$ and since $E$ is discrete, we have that $\Phi(A)$ is finitely nonzero on $E$. Let $\left\{e_{i}\right\}_{i=1}^{n}$ be the set of points $e$ in $E$ such that $\Phi(A)(e) \neq 0$. Then, for $x \in H, A x=\sum_{E}(A x, e) e=$ $\sum_{E}(x, e)(A e, e) e$ (see for example the proof of Theorem 4.1) Hence $A x=\sum_{i=1}^{n}\left(x, e_{i}\right)\left(A e_{i}, e_{i}\right) e_{i}$ and therefore $A(H) \subset \sum_{i=1}^{n} \oplus N_{i}$, where $e_{i} \in N_{i}$, a minimal ideal of $H$. Since each $N_{i}$ is one-dimensional, we have that the range of $A$ is finite dimensional and hence $A \in I_{0}(H)$. Therefore, since each of $I_{0}(H)$ and $\mathscr{C}(H)$ is closed relative to the operator norm, we have that $\mathscr{C}_{\infty}(H) \subset I_{0}(H) \cap \mathscr{C}(H)$. Let $B \in I_{0}(H) \cap \mathscr{C}(H)$, and we can assume that $B=B^{*}$. Thus $B$ is a bounded self-adjoint operator which belongs to the $W^{*}$-algebra $\mathscr{C}(H)$, and since $I \in \mathscr{C}(H)$, we have that $P(a) \in \mathscr{C}(H)$ for all a real, where $P(a)$ is the spectral function of $B$, [2, 448]. Further, $B \in I_{0}(H)$ implies that $B=\sum_{k=1}^{\infty} a_{k} P_{k}$ where $P_{k}=P\left(a_{k}\right)$, each $P_{k}$ is a projection onto a finite dimensional subspace, and $a_{k} \rightarrow 0$ as $k \rightarrow \infty$, [2, 250]. Hence $B=\sum_{k=1}^{\infty} a_{k} P_{k}$ where $P_{k} \in \mathscr{C}(H)$. We will now show that the function on $E$, which maps $e$ to $\left(P_{k} e, e\right)$, is finitely nonzero for each $k$. Let $e \in E$ such that $P_{k} e \neq 0$. By Lemma 4.2, we know that $H=I_{1} \oplus I_{2}$ where $P_{k}=P_{I_{2}}, I_{1} I_{2}=(0), I_{1}$ is an ideal and $I_{2}$ is a subalgebra. Since $e \in E \subset H$, there exists $e_{1} \in I_{1}$, $e_{2} \in I_{2}$ and an irreducible self-adjoint idempotent, $e_{a}$ such that $e=$ $e_{1}+e_{2}=e_{a} /\left\|e_{a}\right\|$. Therefore, $e_{1}+e_{2}=\left\|e_{a}\right\| e e=\left\|e_{a}\right\|\left(e_{1} e_{1}+e_{2} e_{2}\right)$. It follows that $\left\|e_{a}\right\| e_{1}$ and $\left\|e_{a}\right\| e_{2}$ are self-adjoint idempotents and 
$\left(\left\|e_{a}\right\| e_{1}\right)\left(\left\|e_{a}\right\| e_{2}\right)=0$. However, $e_{a}=\left\|e_{a}\right\| e_{1}+\left\|e_{a}\right\| e_{2}$ and since $e_{a}$ is irreducible, $\left\|e_{a}\right\| e_{1}=0$ or $\left\|e_{a}\right\| e_{2}=0$ and therefore $e_{1}=0$ or $e_{2}=0$. It was assumed that $P_{k} e \neq 0$ and $P_{k} e=e_{2}$, so that $e_{1}=0$. Therefore $\left\{e \in E: P_{k} e \neq 0\right\}$ is a subset of $P_{k}(H)$, which is finite dimensional. Thus $P_{k} e$ is finitely nonzero and hence $\Phi\left(P_{k}\right)(e)=\left(P_{k} e, e\right)$ is finitely nonzero. This gives us that $P_{k} \in \mathscr{C}_{0}(H)$ for each $k$ and hence $B$ is an element of the operator norm closure of $\mathscr{C}_{0}(H)$ which is $\mathscr{C}_{\infty}(H)$.

5. Commutative $\boldsymbol{H}^{*}$-algebras, The study of commutative $H^{*}$ algebras is best motivated by $L^{2}(G)$, the convolution algebra of squareintegrable functions on the compact abelian topological group $G$. It seems natural to ask in what sense does $L^{2}(G)$ determine the group $G$. For example, it is known, [4, 92], that if there is an isomorphism from $L^{1}(G)$ onto $L^{1}(H), G$ and $H$ compact abelian topological groups, with norm less then or equal to one, then $G$ and $H$ are isomorphic. The space $L^{2}(G)$ is not as closely related to the group structure in that it . is possible to have nonisomorphic groups whose spares of square-integrable functions are isometric and *-algebra isomorphic. For example, the correspondence

$$
(a, b, c, d) \rightarrow\left(a, \frac{(c+d)+(c-d) i}{2}, b, \frac{(c+d)+(d-c) i}{2}\right)
$$

is an isometric *-algebra isomorphism between the respective spaces of square-integrable functions of the Klein 4-group and the cyclic group on four elements. We will show that $L^{2}(G)$ and $L^{2}(H)$ are isometric *-algebra isomorphic if and only if there is a one-to-one correspondence between $\hat{G}$ and $\hat{H}$, the respective character groups of $G$ and $H$.

Theorem 5.1. Let $H_{i}(i=1,2)$ be commutative $H^{*}$-algebras such that all the irreducible self-adjoint idempotents of $H_{i}$ have norm $k_{i}$. There is a mapping from $H_{1}$ onto $H_{2}$ which is a ${ }^{*}$-algebra isomorphism and a topological mapping if and only if $H_{1}$ and $H_{2}$ have the same dimension, as Hilbert spaces.

Proof. Denote by $E_{1}$ and $E_{2}$ the collections of irreducible self-adjoint idempotents of $H_{1}$ and $H_{2}$. Suppose that $E_{1}$ and $E_{2}$ are in one-to-one correspondence and for $e_{a} \in E_{1}$, denote the corresponding member of $E_{2}$ by $f_{a}$. We may now assume that $E_{1}$ and $E_{2}$ are indexed by the same set. For $x \in H_{1}$, we have that $x=\sum_{a}\left(x, e_{a}\right) e_{a} / k_{1}^{2}$, where $k_{1}=\left\|e_{a}\right\|$ for all $e_{a} \in E_{1}$. Define $\theta$ on $H_{1}$ by $\theta(x)=\sum_{a}\left(x, e_{a}\right) f_{a} / k_{1}^{2}$ and it is clear that $\theta$ is linear and into $H_{2}$. Notice that $\left.\left(x y, e_{a}\right)=\left(x\left[\sum_{b}\left(y, e_{b}\right) e_{b} / k_{1}^{2}\right]\right), e_{a}\right)=$ $\left(y, e_{a}\right)\left(x, e_{a}\right) / k_{1}^{2}$ for $x, y \in H_{1}$ and $e_{a} \in E_{1}$. Hence

$$
\theta(x) \theta(y)=\left[\sum_{a}\left(x, e_{a}\right) f_{a} / k_{1}^{2}\right]\left[\sum_{b}\left(y, e_{b}\right) f_{b} / k_{1}^{2}\right]=\sum_{a}\left(x, e_{a}\right)\left(y, e_{a}\right) f_{a} / k_{1}^{4}=\theta(x y)
$$


and $\theta$ is a homomorphism. It follows easily that $\theta$ is onto, preserves involution and satisfies $\|\theta(x)\|=\left(k_{2} / k_{1}\right)\|x\|$. We have constructed the desired mapping.

For the converse, suppose the mapping $\theta$ is given. Since $\theta$ and $\theta^{-1}$ are isomorphisms, it readily follows that $\theta\left(e_{a}\right)$ is an irreducible self-adjoint idempotent for $H_{2}$ and thus is some member of $E_{2}$, say $f_{a}$. Hence the restriction of $\theta$ to $E_{1}$ is a one-to-one mapping $E_{1}$ into $E_{2}$. Upon applying a dual argument to $\theta^{-1}$; we can conclude that the restriction of $\theta$ to $E_{1}$ is the desired one-to-one correspondence.

REMARK. In the case that $k_{1}=k_{2}$, the proof given above shows that $\theta$ is an isometry.

THEOREM 5.2. Let $H$ be a commutative $H^{*}$-algebra in which all the irreducible self-adjoint idempotents have norm $k$. There is a compact abelian topological group $G$ and a mapping $\theta$ from $H$ onto $L^{2}(G)$ which is a topological *-algebra isomorphism.

Proof. Let $E_{d}$ denote $E$ (the set of irreducible self-adjoint idempotents of $H$ ) endowed with the discrete topology and any abelian group structure. It is always possible to introduce on $E$ an abelian group structure by embedding $E$ in the direct sum (weak direct product) of the integers modulo two, where the index set ranges over $E$. Let $G$ be the group of continuous characters on $E_{d}$. Then $G$ is a compact abelian topological group whose character group is $E_{d}$ and $L^{2}(G)$ is a commutative $H^{*}$-algebra with regular maximal ideal space $E_{d}$. The conclusion now follows easily from Theorem 5.1.

REMARK. If $k=1$, then the mapping is also an isometry.

THEOREM 5.3. If $G$ and $H$ are compact abelian topological groups, then $L^{2}(G)$ and $L^{2}(H)$ are isometric *-algebra isomorphic if and only if there is a one-to-one correspondence between $\hat{G}$ and $\hat{H}$, the respective character groups of $G$ and $H$.

Proof. This theorem can be obtained from Theorem 5.1 by taking $L^{2}(G)=H_{1}, L^{2}(H)=H_{2}, \widehat{G}=E_{1}, \hat{H}=E_{2}$ and $k_{1}=k_{2}=1$.

The author wishes to express his gratitude to Professor H. S. Collins for his many helpful suggestions.

\section{REFERENCES}

1. L. H. Loomis, An introduction to abstract harmonic analysis, Van Nostrand, Princeton, 1953.

2. M. A. Naimark, Normed rings, P. Noordhoff, Gronigen, The Netherlands, 1960.

3. C. E. Rickart, General theory of Banach algebras, Van Nostrand, Princeton, 1960. 
4. W. Rudin, Fourier analysis on groups, Interscience, New York, 1962.

5. Ju-Kwei Wang, Multipliers of commutative Banach algebras, Pacific J. Math. 11 (1961), 1131-1149.

6. J. G. Wendel, Left centralizers and isomorphisms of group algebras, Pacific J. Math. 2 (1952), 251-261.

LOUISIANA State UNIVERSITY

UNIVERSITY OF KENTUCKY 



\section{PACIFIC JOURNAL OF MATHEMATICS}

\section{EDITORS}

\section{H. SAMELSON}

Stanford University

Stanford, California

R. M. Blumenthal

University of Washington

Seattle, Washington 98105

\author{
*J. DugundJI \\ University of Southern California \\ Los Angeles, California 90007 \\ RICHARD ARENS \\ University of California \\ Los Angeles, California 90024
}

\section{ASSOCIATE EDITORS}
E. F. BECKENBACH
B. H. NeUManN
F. WolF
K. YosIDA

\section{SUPPORTING INSTITUTIONS}

UNIVERSITY OF BRITISH COLUMBIA
CALIFORNIA INSTITUTE OF TECHNOLOGY
UNIVERSITY OF CALIFORNIA
MONTANA STATE UNIVERSITY
UNIVERSITY OF NEVADA
NEW MEXICO STATE UNIVERSITY
OREGON STATE UNIVERSITY
UNIVERSITY OF OREGON
OSAKA UNIVERSITY
UNIVERSITY OF SOUTHERN CALIFORNIA

UNIVERSITY OF BRITISH COLUMBIA

UNIVERSITY OF CALIFORNIA

MONTANA STATE UNIVERSITY

NEW MEXICO STATE UNIVERSITY

OREGON STATE UNIVERSITY

OSAKA UNIVERSITY

UNIVERSITY OF SOUTHERN CALIFORNIA

\author{
STANFORD UNIVERSITY \\ UNIVERSITY OF TOKYO \\ UNIVERSITY OF UTAH \\ WASHINGTON STATE UNIVERSITY \\ UNIVERSITY OF WASHINGTON \\ AMERICAN MATHEMATICAL SOCIETY \\ CHEVRON RESEARCH CORPORATION \\ TRW SYSTEMS \\ NAVAL ORDNANCE TEST STATION
}




\section{Pacific Journal of Mathematics \\ Vol. 17, No. $1 \quad$ January, 1966}

Carlos Jorge Do Rego Borges, On stratifiable spaces ................ 1

Felix Earl Browder, Topological methods for non-linear elliptic equations of

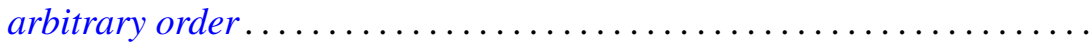

Gustave Choquet, Harry Corson and Victor Klee, Exposed points of convex

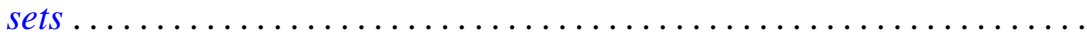

Phillip Emig, Remarks on the defect sum for a function meromorphic on an open Riemann surface ................................ 45

Ruth Goodman, A certain class of polynomials .................. 57

Sidney (Denny) L. Gulick, The bidual of a locally multiplicatively-convex

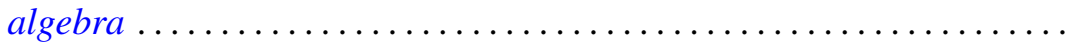

Eugene Carlyle Johnsen, Integral solutions to the incidence equation for finite projective plane cases of orders $n \equiv=2(\bmod 4) \ldots \ldots \ldots \ldots . .67$

Charles N. Kellogg, Centralizers and $H^{*}$-algebras .................. 121

Michael Lodato, On topologically induced generalized proximity relations. II .......................................... 131

P. H. Maserick, Half rings in linear spaces ..................... 137

Kathleen B O'Keefe, On a problem of J. F. Ritt .................... 149

Galen Lathrop Seever, Nonnegative projections on $C_{0}(X) \ldots \ldots \ldots \ldots$

Lawrence A. Shepp, Gaussian measures in function space ............ 167

Robert Charles Thompson, Classes of definite group matrices ........... 175 